\title{
Scrub typhus associated with hemophagocytic lymphohistiocytosis: A report of six pediatric patients
}

\author{
YINGKANG JIN $^{1}$, LI HUANG ${ }^{2}$, HUIFENG FAN ${ }^{1}$, GEN LU $^{1}$, YI XU ${ }^{3}$ and ZHIYUAN WU ${ }^{2}$ \\ ${ }^{1}$ Department of Respiratory Infection; ${ }^{2}$ Pediatric Intensive Care Unit; ${ }^{3}$ Department of Infectious Diseases, \\ Guangzhou Women and Children's Medical Center, Guangzhou Medical University, \\ Guangzhou, Guangdong 510120, P.R. China
}

Received March 24, 2015; Accepted June 14, 2016

DOI: $10.3892 /$ etm.2016.3668

\begin{abstract}
Hemophagocytic lymphohistiocytosis (HLH) is a life-threatening immune disorder that may be inherited or secondary to infection, malignancy or rheumatological disease. The aim of the present study was to highlight the clinical features of scrub typhus-associated HLH in children. A retrospective study was performed on 6 pediatric patients with scrub typhus-associated HLH. For each patient, medical records were reviewed and analyzed, and demographic, clinical and laboratory data and outcomes were collected. The duration of fever prior to admission ranged between 4 and 12 days. All patients exhibited persistent or intermittent fever, eschar, hepatosplenomegaly and lymphadenopathy at the time of diagnosis. Five patients experienced acute respiratory distress syndrome and disseminated intravascular coagulation (DIC) during hospitalization. Thrombocytopenia was detected in all patients with cytopenia involving two or three cell types, simultaneously. Coagulopathy with prolonged prothrombin time and/or activated partial thromboplastin time were noted in all patients. Markedly elevated serum ferritin levels $(>1,500 \mu \mathrm{g} / \mathrm{ml})$ were identified in all patients. Elevated lactate dehydrogenase $(>1,000 \mathrm{U} / \mathrm{l})$ was detected in $4(66.7 \%)$ patients and elevated alanine aminotransferase was exhibited by $5(83 / 3 \%)$ patients. Lung infiltrates and consolidation were the most common imaging findings. Only 1 patient succumbed, with DIC and multi-organ failure. Of the survivors, 1 patient was lost to follow-up, and the remaining patients are in remission with excellent general health, to date. In conclusion, HLH should be considered in severe pediatric cases of scrub typhus. Upon the early recognition of this syndrome, prompt and supportive treatment in the pediatric intensive care unit are vital.
\end{abstract}

Correspondence to: Professor Gen Lu, Department of Respiratory Infection, Guangzhou Women and Children's Medical Center, Guangzhou Medical University, 9 Jinsui Road, Guangzhou, Guangdong 510120, P.R. China

E-mail: genlucn@163.com

Key words: scrub typhus, hemophagocytic lymphohistiocytosis, severe disease, children

\section{Introduction}

Hemophagocytic lymphohistiocytosis (HLH) is a life-threatening immune disorder that is characterized by fever, massive hepatosplenomegaly, pancytopenia, hypertriglyceridemia, hypofibrinogenemia and seizures (1-3). The disease may be inherited (primary or familial) or secondary to infection, malignancy or rheumatological disease, and can occur at any age (3). Infection-associated HLH has prominent links with various viral, bacterial, fungal and parasitic infections, including Epstein-Barr virus (EBV), influenza, typhoid, tuberculosis, leishmaniasis and scrub typhus (2). Scrub typhus, a febrile rickettsial disease, is often caused by Orientia tsutsugamushi, which is endemic in various Asian countries, including China, India, Korea and other areas in southeast Asia (4-6). Its clinical manifestations are non-specific, as they typically include fever, skin rash, myalgia, gastrointestinal disturbances and lymphadenopathy (4). Nevertheless, the majority of reported cases of scrub typhus have shown prompt improvement with anti-rickettsial treatment. To date, previous examples of scrub typhus-associated HLH in children have been described only as single case reports $(5,6)$; therefore, the precise distribution and spectrum of scrub typhus associated with this disorder remains largely unknown.

The present study reports HLH and acute respiratory distress syndrome (ARDS) occurring in 6 pediatric patients as a complication of scrub typhus.

\section{Patients and methods}

Patients. The clinical data of 6 pediatric patients (4 boys and 2 girls; age range, 8 months to 11 years) that were admitted to Guangzhou Women and Children's Medical Center, Guangzhou Medical University (Guangzhou, China) with scrub typhus complicated with HLH and ARDS between 2010 and 2014 were retrospectively reviewed. A history of playing outdoors in the summer or fall in rural areas where scrub typhus was endemic, high fever $\left(>38.5^{\circ} \mathrm{C}\right)$, lymphadenopathy, splenomegaly and eschar lesions on covered areas (scalp, axilla, groin or genitalia) were identified as markers of suspected scrub typhus on admission. Laboratory diagnosis of scrub typhus was confirmed using a Weil-Felix agglutination test or/and the detection of elevated 
serum antibodies [O.tsutsugamushi immunoglobulin M (IgM)] using immunofluorescence, as previously described (7). Each patient was diagnosed with HLH based on criteria proposed by the Histiocyte Society in 2004 (8).

The present study was conducted in accordance with the Declaration of Helsinki, and was approved by the Ethics Committee of Guangzhou Medical University. Written informed consent was obtained from the guardians of all participants.

Diagnostic methods. For each patient, clinical and laboratory data, and details of the disease course, treatment and outcomes were collected and analyzed. Blood and bone marrow samples were cultured for bacteria, fungi and viruses. In addition, serologic and/or nucleic acid-based diagnostic testing for EBV, cytomegalovirus (CMV) and parvovirus B19 was conducted for all patients, as previously described (7). Serological testing for human immunodeficiency virus, hepatitis B and C, mycoplasma pneumonia and TORCH toxoplasma [which includes toxoplasmosis, other (syphilis, varicella-zoster and parvovirus B19) were performed using the ELISA method. A bone marrow sample, obtained by needle aspiration, was obtained from each of the patients, and routine serological and hematological analyses were performed using standard procedures. Hepatosplenomegaly and lymphadenopathy were diagnosed via physical examination and ultrasonography.

\section{Results}

Clinical features. All patients had a history of playing outdoors in the summer or fall in rural areas where scrub typhus was endemic. Only 1 patient had been diagnosed with scrub typhus prior to admission. Three patients were diagnosed with pneumonia or fever of unknown origin prior to admission to hospital, and two patients had been suspected of having HLH. As a result of an elevated peripheral atypical lymphocyte count (15\%), 1 patient was suspected to have EBV-associated HLH; however, this was contradicted by negative results for EBV capsid antigen IgM antibody and EBV nuclear antibody, and a low EBV load. The duration of fever prior to admission ranged from 4 to 12 days. All patients exhibited persistent or intermittent fever, eschar (Fig. 1), hepatosplenomegaly and lymphadenopathy at the time of diagnosis. Features of the 6 patients whose data included manifestations of disease, identified on admission or developed during hospitalization, are presented in Table I. Gastrointestinal discomfort manifesting with vomiting, diarrhea and abdominal pain was present in 5 patients on admission. Seizure, hematuria and jaundice were each present in 1 patient. In addition, 5 patients experienced complicating ARDS and disseminated intravascular coagulation (DIC) during hospitalization, and an intracranial hemorrhage was identified in 1 patient.

Laboratory data. Serological results for human immunodeficiency virus, parvovirus B19, hepatitis B and C, CMV, EBV, mycoplasma pneumonia and TORCH toxoplasma [which includes toxoplasmosis, other (syphilis, varicella-zoster and parvovirus B19), rubella, CMV and herpes infections] were negative in all patients. Two patients (cases 3 and 6) were diagnosed with infection with Staphylococcus epidermidis

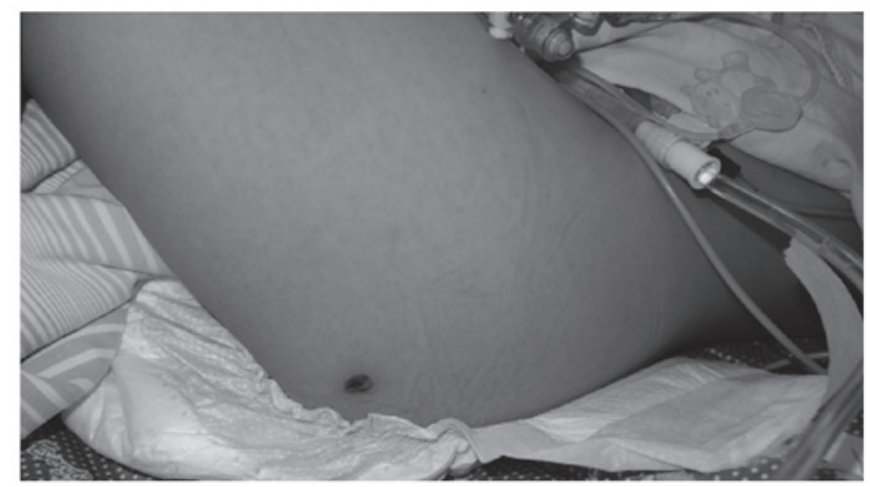

Figure 1. A necrotic eschar identified in the vicinity of the left buttock of Case 2 .

and Hemophilus influenzae, respectively. Viral serologic results revealed the presence of respiratory syncytial virus in 1 patient (case 4). The laboratory findings at the time of diagnosis are presented in Table I. Thrombocytopenia was detected in all patients with cytopenia involving two or three cell types, simultaneously. Coagulopathy with prolonged prothrombin time (PT) and/or activated partial thromboplastin time were noted in all patients. Markedly elevated serum ferritin $(>1,500 \mu \mathrm{g} / \mathrm{ml})$ values were observed in all patients. Elevated lactate dehydrogenase $(>1,000 \mathrm{U} / \mathrm{l})$ was detected in $4(66.7 \%)$ patients and elevated alanine aminotransferase was exhibited by $5(83 / 3 \%)$ patients. According to the diagnostic criteria for HLH proposed by the Histiocyte Society (8), hypertriglyceridemia is diagnosed when the serum triglyceride is $\geq 3 \mathrm{mmol} / \mathrm{l}$ (normal range, 0.23-1.70 $\mathrm{mmol} / \mathrm{l}$ ) and hypofibrinogenemia is diagnosed when serum fibrinogen is $\leq 1.5 \mathrm{~g} / \mathrm{l}$ (normal range, $2.00-4.00 \mathrm{~g} / \mathrm{l}$ ). During the early stage of the disease, hypertriglyceridemia and hypofibrinogenemia were observed in $5(83.3 \%)$ and $4(66.7 \%)$ patients, respectively. During the late stage of the disease, hypertriglyceridemia and hypofibrinogenemia were observed in all 6 patients. Hemophagocytosis (Fig. 2) was identified in all patient bone marrow biopsies. Lung infiltrates and consolidation were the most common features observed by imaging (Fig. 3).

Treatments and outcomes. As shown in Table II, all patients received anti-rickettsial antibiotics following the diagnosis of scrub typhus. Anti-rickettsial antibiotic treatment included azithromycin (10 mg/kg; once daily; Pfizer, Inc., New York, NY, USA) for three consecutive days every week for 2-3 weeks, and doxycycline hyclate $(2.2 \mathrm{mg} / \mathrm{kg}$ twice daily, which was altered to once daily following normothermia; Hainan Kangli Pharmaceutical Co., Ltd., Hainan, China) for 7-10 days if the patient was $>7$ years old. All patients were administered intravenous immunoglobulin (IVIG) and mechanical ventilation due to complications related to ARDS, and 5 patients were administered systemic steroid therapy (intravenous $2 \mathrm{mg} / \mathrm{kg}$ methylprednisolone 1-2 times/day for 3-7 consecutive days; Pfizer, Inc.). Plasma exchange and continuous renal replacement therapy (CRRT) was performed on 1 patient. One patient succumbed to DIC and multiple organ failure (MOF) on day 7 of hospitalization. Five patients showed remission. The duration of hospital stay ranged between 7 and 24 days. To date, of the 5 survivors, 1 patient has been lost to 


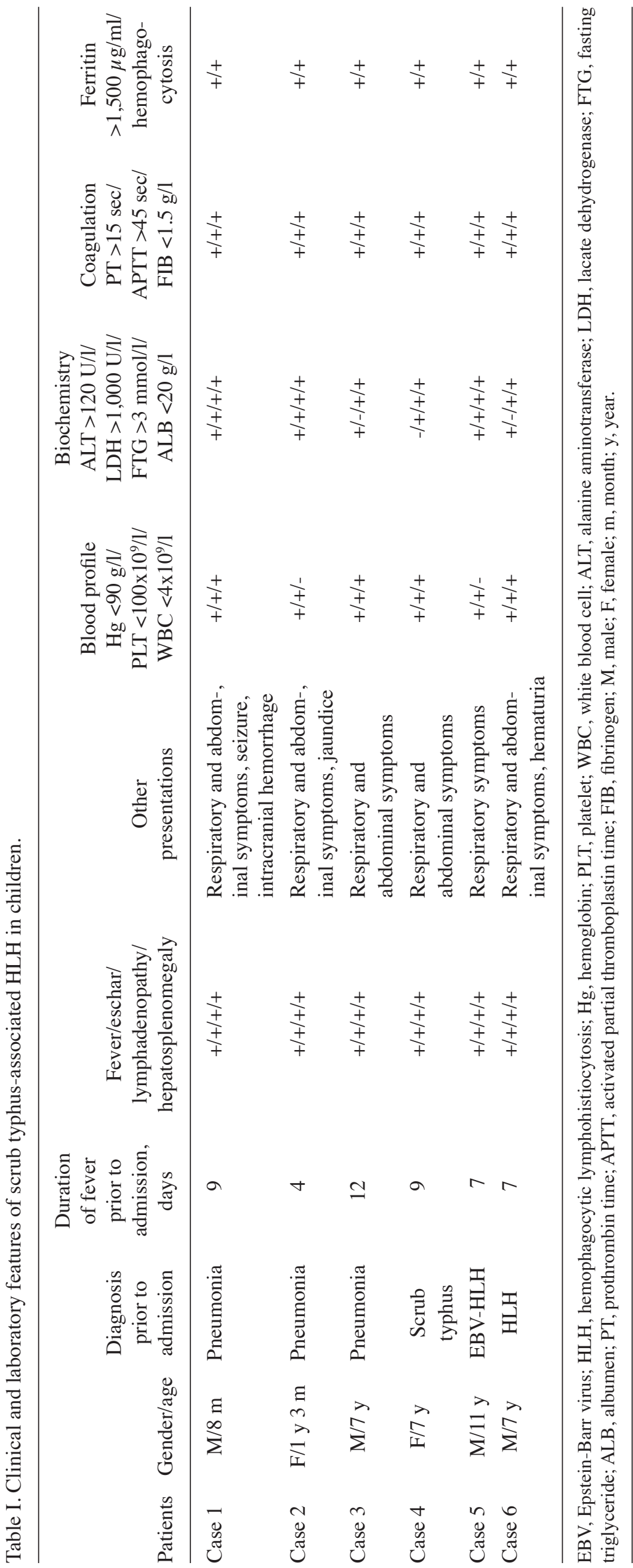


Table II. Treatments and outcomes of scrub typhus associated HLH in children.

\begin{tabular}{lcccclcl}
\hline Patient & $\begin{array}{c}\text { IVIG/ } \\
\text { steroids }\end{array}$ & $\begin{array}{c}\text { Anti-rickettsial } \\
\text { antibiotics }\end{array}$ & $\begin{array}{c}\text { Mechanical } \\
\text { ventilation }\end{array}$ & $\begin{array}{c}\text { Other } \\
\text { supportive therapy }\end{array}$ & Complications & $\begin{array}{c}\text { Hospital } \\
\text { stay (days) }\end{array}$ & Outcome \\
\hline Case 1 & $+/+$ & Azithromycin & $+(9$ days) & Blood products & ARDS, DIC, MOF & 7 & DNS \\
Case 2 & $+/+$ & Azithromycin & $+(10$ days) & Blood products & ARDS, DIC & 22 & Remission \\
Case 3 & $+/+$ & Doxycycline & $+(11$ days $)$ & Blood products & ARDS & 21 & Remission \\
Case 4 & $+/+$ & Doxycycline & $+(14$ days $)$ & Blood products & ARDS, DIC & 24 & Remission \\
Case 5 & $+/-$ & Doxycycline & - & Blood products & DIC & 14 & Remission \\
Case 6 & $+/+$ & Doxycycline & $+(9$ days $)$ & Blood products, & ARDS, DIC & 16 & Remission \\
& & & & PE and CRRT & & &
\end{tabular}

None of the patients were treated according to the 2004-HLH protocol. HLH, hemophagocytic lymphohistiocytosis; IVIG, intravenous immunoglobulin; ARDS, acute respiratory distress syndrome; DIC, disseminated intravascular coagulation; MOF, multiple organ failure; DNS, did not survive; PE, plasma exchange; CRRT, continuous renal replacement therapy.

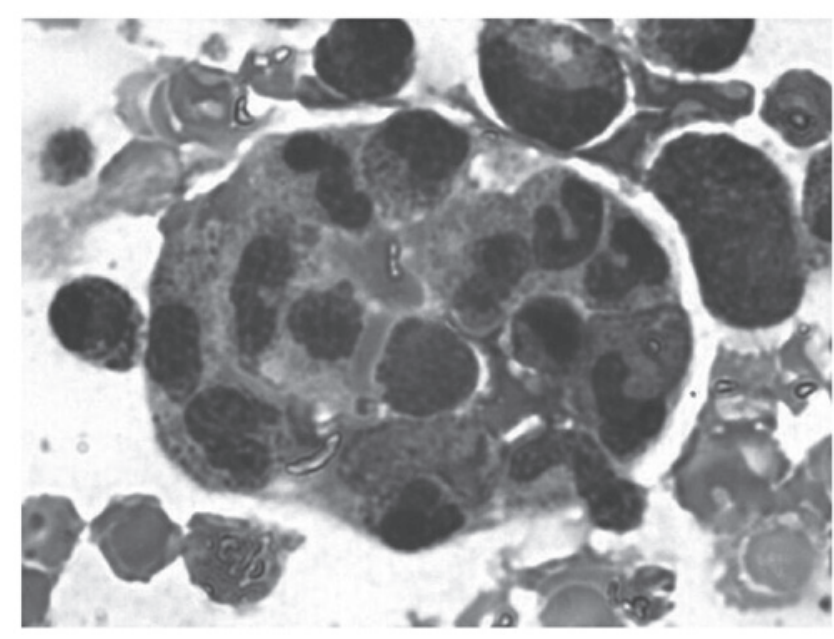

Figure 2. Histiocytes with phagocytosed blood cells identified by bone marrow biopsy on Case 5 (magnification, x1,000).

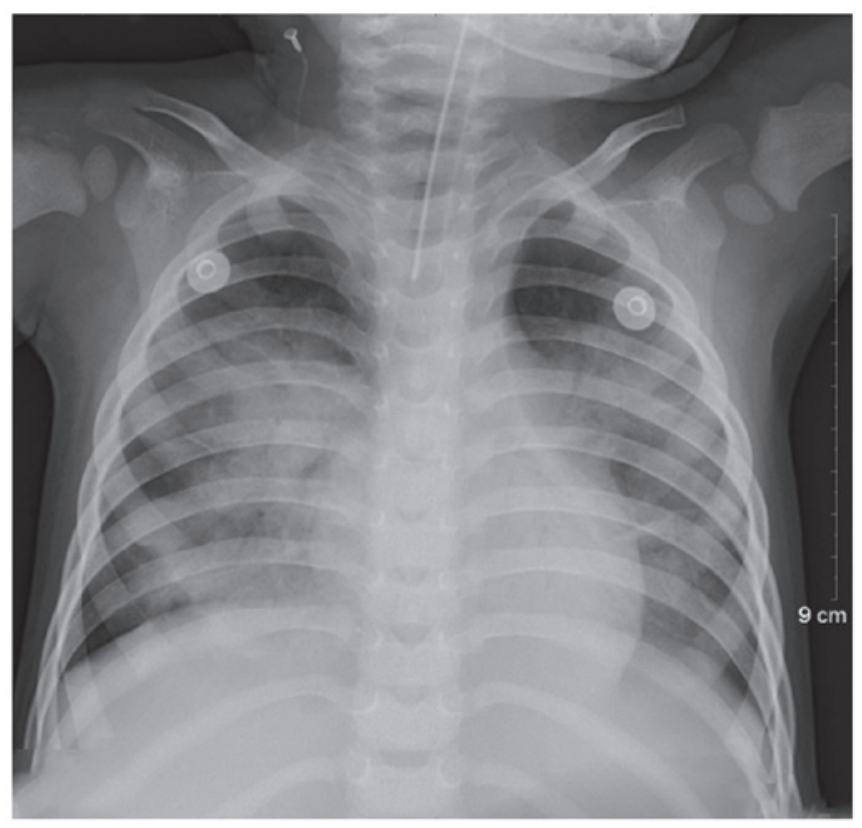

Figure 3. Chest X-ray revealed bilateral ground-glass opacities, indicative of acute respiratory distress syndrome, in Case 2. follow-up, and the remaining patients are in remission with excellent general health.

\section{Discussion}

Scrub typhus, a febrile disease caused by rickettsial infection from O. tsutsugamushi, is common in Asia-Pacific countries, including China, India, Korea and other areas in southeast Asia $(4,9)$. Scrub typhus is manifested clinically by a high fever, rash, splenomegaly and lymphadenopathy, which can disappear in a few days (4). However, severe manifestations caused by complications of MOF, ARDS, shock and DIC may also occur $(6,10,11)$. The basic pathology of scrub typhus is small vessel vasculitis, particularly of the lung, heart, brain and kidney (11). The initial clinical symptoms of scrub typhus are non-specific, and patients often present to the physician with the appearance of a common fever of unknown origin. There is a high chance of misdiagnosis of the disease at the early stage; in the current study, 5 of 6 patients were not diagnosed with scrub typhus on admission. A combination of the following clinical manifestations can aid in the diagnosis of the disease: Fever of unknown origin, hepatosplenomegaly and regional lymphadenopathy with eschar lesions on covered areas of skin.

Patients with severe complications have a reported mortality rate as high as $30 \%$ without proper treatment (10). Recent reports have suggested that the cytokine storm associated with the immune response to O. tsutsugamushi infection may be involved in the pathogenesis of complicated scrub typhus (12-14). Once the cytokine cascade has been triggered, the condition can become a potentially life-threatening illness. In the present study, the majority of the patients experienced ARDS and DIC, and satisfied the diagnostic criteria of HLH. Therefore, the possible diagnosis of secondary HLH should also been considered alongside scrub typhus. Scrub typhus associated-HLH in children is rarely reported. The formal guidelines for evaluating patients with suspected $O$. tsutsugamushi infection-associated HLH have not been established. Clinicians are recommended to consider scrub typhus as a possible etiology while handling cases of HLH in the epidemic areas. 
Although hemophagocytosis is absent in the initial bone marrow aspirate in the majority of cases and thrombocytopenia is not a common phenomenon in patients with scrub typhus $(3,15)$, which was observed upon diagnosis for all of the patients in the present study, it was noted that all serum ferritin expression levels were markedly increased to $>1,500 \mu \mathrm{g} / \mathrm{ml}$, and lactate dehydrogenase expression levels were markedly elevated $(>1,000 \mathrm{U} / 1)$. Serum ferritin is a particularly sensitive indicator that reflects the disease activity, and can be used as an indicator for curative effect and the prognosis of HLH (16). Markedly elevated serum ferritin expression levels may occur due to inflammatory cytokines promoting ferritin synthesis (16). The clinical and laboratory findings in the current study suggest that the activation of monocyte/macrophage cell-induced inflammatory cytokines develops during scrub typhus infection and reflects the severity of the disease in the patients. It is possible that a number of the patients may have had primary, not secondary, HLH, because genetic testing was not performed. One patient had an elevated peripheral atypical lymphocyte count $(15 \%)$, demonstrating that scrub typhus can result in infectious mononucleosis.

Pediatric therapeutic strategies for scrub typhus-associated HLH are not well established in the literature. As aforementioned, profound hypercytokinemia serves a key role in the pathophysiology of severe HLH. Controlling hypercytokinemia is the first step in saving a patient's life $(16,17)$. The patients in the present study were not treated according to the 2004-HLH protocol (consisting of etoposide, cyclosporine and dexamethasone); they were treated with anti-rickettsial antibiotics and immunomodulatory therapy (IVIG and/or corticosteroids) alongside supported treatment (blood products and mechanical ventilation). In addition, plasma exchange and CRRT were performed in 1 patient. Steroids are frequently used in combination with IVIG to treat cases of virus-associated HLH. Hyperinflammation can be treated with corticosteroids, which are cytotoxic for lymphocytes and inhibit the expression of cytokines and the differentiation of dendritic cells $(3,15)$. Furthermore, IVIG is effective in hyperferritinemia (18). Previous reports suggest that infection-associated HLH has a severe clinical course and a poor prognosis, emphasizing the importance of early diagnosis and prompt therapy to shorten the disease course, and reduce mortality and mortality $(16,19,20)$. The prognosis of scrub typhus-associated HLH is unknown. In the current study, one patient (16.7\%) succumbed to DIC and MOF, which demonstrated that there is a relatively low rate of mortality resulting from scrub typhus-associated HLH. The results demonstrated that anti-rickettsial treatment and the use of immunoregulatory therapy may be effective in the treatment of the inflammatory cytokine storm experienced in children with scrub typhus-associated HLH. The laboratory results of patient 6 improved markedly following plasma exchange and CRRT. These treatments can remove large quantities of cytokines from the plasma and may be a beneficial method of treatment. However, there were too few patients included the current study to draw any conclusions, and further studies are required to confirm a therapeutic strategy for scrub typhus-associated HLH.

ARDS and DIC are serious and very high-frequency complications of HLH in the context of scrub typhus compared with other causes. This can be explained by vasculitis caused by the pathogen itself, and is exacerbated by the induced inflammatory cytokine storm, since most cases were improved following treatment with anti-rickettsial and immunosuppressive therapy. In the current study, only 1 patient, an 8-month old boy, succumbed to refractory bleeding and MOF despite receiving aggressive treatment, which included the administration of anti-rickettsial antibiotics and immunomodulatory therapies (IVIG and corticosteroid) with additional supporting treatments (blood products and mechanical ventilation). This patient had a 9-day delayed diagnosis and experienced complications with MOF, DIC and septic shock on admission. Delayed diagnosis (and delayed initial anti-rickettsial treatment), multiple severe complications and young age may be the primary causes of mortality. It may be suggested that an early diagnosis and appropriate treatment may improve the clinical outcomes and reduce the complications associated with HLH in children.

In conclusion, the present study demonstrates that HLH should be considered as a diagnosis in severe pediatric cases of scrub typhus. The majority of the patients in the current study had a positive outcome. Early recognition of this syndrome, prompt treatment and supportive treatment in a pediatric intensive care unit are key factors for survival. Further studies are required to confirm a therapeutic strategy for scrub typhus-associated HLH; anti-rickettsial antibiotic treatment combined with an immunomodulatory treatment (steroids and IVIG) may be beneficial in such cases, instead of treatment according to the 2004-HLH protocol. In addition, plasma exchange and CRRT may be beneficial treatments for severe scrub typhus-associated HLH; however, more data are required to clarify the optimal treatment protocol and the expected outcomes.

\section{References}

1. Verbsky JW and Grossman WJ: Hemophagocytic lymphohistiocytosis: Diagnosis, pathophysiology, treatment and future perspectives. Ann Med 38: 20-31, 2006.

2. Domachowske JB: Infectious triggers of hemophagocytic syndrome in children. Pediatr Infect Dis J 25: 1067-1068, 2006.

3. Janka GE: Familial and acquired hemophagocytic lymphohistiocytosis. Annu Rev Med 63: 233-246, 2012.

4. Henter JI, Horne A, Aricó M, Egeler RM, Filipovich AH, Imashuku S, Ladisch S, McClain K, Webb D, Winiarski J and Janka G: HLH-2004: Diagnostic and therapeutic guidelines for hemophagocytic lymphohistiocytosis. Pediatr Blood Cancer 48: 124-131, 2007.

5. Han DK, Baek HJ, Shin MG, Kim JW, Kook H, Hwang TJ: Scrub typhus-associated severe hemophagocytic lymphohistiocytosis with encephalomyelitis leading to permanent sequelae: a case report and review of the literature. J Pediatr Hematol Oncol. 34:531-533, 2012.

6. Kwon HJ, Yoo IH, Lee JW, Chung NG, Cho B, Kim HK and Kang JH: Life-threatening scrub typhus with hemophagocytosis and acute respiratory distress syndrome in an infant. J Trop Pediatr 59: 67-69, 2013.

7. Koraluru M, Bairy I, Varma M, and Vidyasagar S: Diagnostic validation of selected serological tests for detecting scrub typhus. Microbiol Immunol 59: 371-374, 2015.

8. Jeong YJ, Kim S, Wook YD, Lee JW, Kim KI and Lee SH: Scrub typhus: Clinical, pathologic and imaging findings. Radiographics 27: 161-172, 2007.

9. Kamarasu K, Malathi M, Rajagopal V, Subramani K, Jagadeeshramasamy D and Mathai E: Serological evidence for wide distribution of spotted fevers \& typhus fever in Tamil Nadu. Indian J Med Res 126: 128-130, 2007.

10. Kim DM, Kim SW, Choi SH and Yun NR: Clinical and laboratory findings associated with severe scrub typhus. BMC Infect Dis 10: 108, 2010. 
11. Lee CS, Hwang JH, Lee HB and Kwon KS: Risk factors leading to fatal outcome in scrub typhus patients. Am J Trop Med Hyg 81: 484-488, 2009.

12. Cascio A, Pernice LM, Barberi G, Delfino D, Biondo C, Beninati C, Mancuso G, Rodriguez-Morales AJ and Iaria C: Secondary hemophagocytic lymphohistiocytosis in zoonoses. A systematic review. Eur Rev Med Pharmacol Sci 16: 1324-1337, 2012.

13. Valsalan R, Kosaraju K, Sohanlal T and Kumar PS: Hemophagocytosis in scrub typhus. J Postgrad Med 56: 301-302, 2010.

14. Chen YC, Chao TY and Chin JC: Scrub typhus-associated hemophagocytic syndrome. Infection 28: 178-179, 2000.

15. Nahum E, Ben-Ari J, Stain J and Schonfeld T: Hemophagocytic lymphohistiocytic syndrome: Unrecognized cause of multiple organ failure. Pediatr Crit Care Med 1: 51-54, 2000

16. Imashuku S: Clinical features and treatment strategies of Epstein-Barr virus-associated hemophagocytic lymphohistiocy tosis. Crit Rev Oncol Hematol 44: 259-272, 2002.
17. Freeman HR and Ramanan AV: Review of haemophagocytic lymphohistiocytosis. Arch Dis Child 96: 688-693, 2011.

18. Emmenegger U, Frey U, Reimers A, Fux C, Semela D, Cottagnoud P, Spaeth PJ and Neftel KA: Hyperferritinemia as indicator for intravenous immunoglobulin treatment in reactive macrophage activation syndromes. Am J Hematol 68: 4-10, 2001

19. Jin YK, Xie ZD, Yang S, Lu G and Shen KL: Epstein-Barr virus-associated hemophagocytic lymphohistiocytosis: A retrospective study of 78 pediatric cases in mainland of China. Chin Med J (Engl) 123: 1426-1430, 2010.

20. Yasunaga H, Horiguchi H, Kuwabara K, Hashimoto H and Matsuda S: Delay in tetracycline treatment increases the risk of complications in Tsutsugamushi disease: Data from the Japanese diagnosis procedure combination database. Intern Med 50: 37-42, 2011. 